\title{
Real-World Long-Term Ivacaftor for Cystic Fibrosis in France: Clinical Effectiveness and Healthcare Resource Utilization
}

\author{
Dominique Hubert - Christophe Marguet - Jacques Benichou • \\ Cynthia DeSouza - Catherine Payen-Champenois - Nils Kinnman • \\ Keval Chandarana $\cdot$ Anne Munck $\cdot$ Isabelle Fajac on behalf of the BRIO Study Group
}

Received: March 8, 2021 / Accepted: April 22, 2021 / Published online: June 8, 2021

(C) The Author(s) 2021, corrected publication 2021

\section{ABSTRACT}

Introduction: Ivacaftor is a cystic fibrosis transmembrane conductance regulator (CFTR) potentiator that has demonstrated clinical

The members of the "on behalf of the BRIO Study Group" was processed under acknowledgements section.

Supplementary Information The online version contains supplementary material available at https:// doi.org/10.1007/s41030-021-00158-5.

D. Hubert $(\varangle) \cdot$ I. Fajac

Respiratory Medicine and National Cystic Fibrosis Reference Center, Cochin Hospital, Assistance Publique-Hôpitaux de Paris, Paris, France e-mail: dominique.hubert@aphp.fr

\section{Marguet}

Pediatric Respiratory Diseases and Cystic Fibrosis Center, Rouen University Hospital, INSERM CIC1404, EA 2656 UNI ROUEN, Normandy University, Rouen, France

e-mail: christophe.marguet@chu-rouen.fr

\section{J. Benichou}

Department of Biostatistics and Clinical Research, Rouen University Hospital and INSERM U 1018,

University of Rouen, Rouen, France

e-mail: jacques.benichou@chu-rouen.fr

C. DeSouza

Independent Consultant, Boston, MA, USA

e-mail: cynthia.desouza4@gmail.com

C. Payen-Champenois

Vertex Pharmaceuticals (France), Paris, France

e-mail: catherine_payen-champenois@vrtx.com benefits in phase 3 trials. We report results from a real-world study (BRIO) to assess the effectiveness of ivacaftor in people with cystic fibrosis (pwCF) in France.

Methods: BRIO was an observational study conducted at 35 centers in France. Both pwCF initiating ivacaftor treatment and those already taking ivacaftor were included and prospectively followed for 24 months. The primary objective was to evaluate the effect of ivacaftor on percent predicted forced expiratory volume in $1 \mathrm{~s} \quad\left(\mathrm{ppFEV}_{1}\right)$; secondary objectives were

\section{N. Kinnman}

Vertex Pharmaceuticals (Europe) Limited, London, UK

e-mail: nils_kinnman@vrtx.com

K. Chandarana

Vertex Pharmaceuticals Incorporated, Boston, MA, USA

e-mail: keval_chandarana@vrtx.com

\section{A. Munck}

Cystic Fibrosis Center, Hôpital Robert Debré, Assistance Publique-Hôpitaux de Paris, Paris, France e-mail: anne.munck1@gmail.com

\section{Fajac}

Physiology Department, AP-HP Centre-Université de Paris, Hôpital Cochin, Paris, France

\section{Fajac}

Université de Paris, Paris, France

e-mail: isabelle.fajac@parisdescartes.fr 
evaluating the effect of ivacaftor on clinical effectiveness, healthcare resource utilization (HCRU), and safety.

Results: A total of 129 pwCF were enrolled; $58.9 \%$ were aged $<18$ years; $64.3 \%$ had a G551D-CFTR allele. Mean age at ivacaftor initiation was 19.1 years (range, 2-64 years); ppFEV $_{1}$ increased by a least squares mean of 8.49 percentage points in the first 6 months and was sustained through 36 months of ivacaftor use. Growth metrics increased during the first 12 months post-ivacaftor and remained stable. The rate of pulmonary exacerbations (PEx) decreased during the 12 months post-ivacaftor compared with the 12 months pre-ivacaftor; estimated rate ratios $(95 \% \mathrm{CI})$ were 0.57 (0.43-0.75) for PEx events and 0.25 (0.13-0.48) for PEx requiring hospitalization. No new safety concerns were identified; no deaths occurred.

Conclusions: The results from this real-world study of ivacaftor usage in France were consistent with prior clinical trial outcomes, confirming the clinical effectiveness of ivacaftor, as well as an associated reduction in HCRU.

Keywords: Adults; Children; Cystic fibrosis; Exacerbation; Ivacaftor; Lung function; Realworld experience

\section{Key Summary Points}

Why carry out this study?

Ivacaftor is a cystic fibrosis transmembrane conductance regulator (CFTR) potentiator developed to treat the underlying cause of CF. Treatment with ivacaftor improves clinical outcomes in people with $\mathrm{CF}$ (pwCF) with certain gating and non-gating CFTR mutations.

Since CF is a lifelong disease, it is important to understand the long-term impact of ivacaftor treatment in a realworld setting.

This observational study aimed to assess the real-world effectiveness and healthcare resource utilization after ivacaftor initiation in pwCF in France.

\section{What was learned from the study?}

At 35 CF centers in France, 129 pwCF were enrolled; $58.9 \%$ were $<18$ years of age. Improvements in lung function, rate of pulmonary exacerbations, nutritional status, and growth metrics were observed with ivacaftor use and were sustained over 36 months of follow-up. Additionally, ivacaftor usage was associated with a reduction in healthcare resource utilization.

The results from this real-world study of ivacaftor use in France were consistent with prior clinical trial outcomes and confirm the clinical effectiveness of ivacaftor.

\section{DIGITAL FEATURES}

This article is published with digital features, including a summary slide, to facilitate understanding of the article. To view digital features for this article go to https://doi.org/10.6084/ m9.figshare.14459532.

\section{INTRODUCTION}

Ivacaftor is a cystic fibrosis transmembrane conductance regulator (CFTR) modulator that potentiates the transport of chloride ions through the CFTR channel [1-3] and was developed to treat the underlying cause of cystic fibrosis (CF). In clinical studies, ivacaftor monotherapy has been demonstrated to improve clinical outcomes in people with CF (pwCF) with certain gating and non-gating mutations $[4,5]$.

The combination of multiple CFTR modulators (e.g., lumacaftor and ivacaftor, tezacaftor and ivacaftor, or elexacaftor, tezacaftor, and ivacaftor) has since broadened the population of pwCF who may benefit and has further improved clinical outcomes [6]. Based on clinical trial results $[7,8]$, ivacaftor was initially granted marketing authorization for the 
treatment of pwCF $\geq 6$ years of age with a G551D-CFTR mutation by the European Medicines Agency (EMA) in 2012 [4]. An extension for other Class III non-G551D mutations (G178R, S549N, S549R, G551S, G1244E, $S 1251 N, S 1255 P$, or G1349D) was granted by EMA in 2014, and France followed with expanded market availability for additional mutations in 2015 [9, 10]. Indications for approved use of ivacaftor and reimbursement for ivacaftor in France have continued to expand since initial approval [11-13], and most recently the indication has been expanded by EMA to pwCF as young as 4 months [13]. The health authorities requested real-world data for all pwCF treated with ivacaftor in France in order to evaluate drug effectiveness and safety in pwCF treated at CF centers in France without constraints of clinical trial inclusion and exclusion criteria. Hence, BRIO (Cystic FiBRosis In Observation) was designed as an observational study to assess real-world effectiveness and healthcare resource utilization (HCRU) after initiation of ivacaftor in pwCF with G551D or non-G551D gating mutations in France.

\section{METHODS}

\section{Observational Study Design and Participants}

BRIO was an observational, noninterventional, multicenter, retrospective, prospective study in France for pwCF treated with ivacaftor. The protocol for this study was developed in consultation among Vertex Pharmaceuticals, health authorities, and a scientific steering committee. PwCF were enrolled from the CF centers network (Société française de la mucoviscidose, filière muco-CFTR). Inclusion criteria included pwCF treated with ivacaftor. Two populations were recruited: pwCF already receiving ivacaftor at study inclusion, and pwCF initiating ivacaftor at study inclusion.

The primary objective was to describe the characteristics of pwCF treated with ivacaftor in a real-world setting and to evaluate the impact of ivacaftor on percent predicted forced expiratory volume in $1 \mathrm{~s}\left(\mathrm{ppFEV}_{1}\right)$. Secondary objectives were to evaluate the impact of ivacaftor on the number of pulmonary exacerbations (PEx); HCRU in terms of hospitalization, intravenous (IV) antibiotics courses, and concomitant symptomatic treatments; nutritional status (body mass index [BMI]) and growth metrics (BMI-for-age $z$ score); and pulmonary microbiology. BMI-for-age $z$ score was calculated for those aged $<20$ years (based on the Centers for Disease Control growth chart from 2000 [14]) because it allows for standardization of BMI to normal populations of children of different ages ( $\leq 20$ years) and both sexes. The occurrence of adverse events (AEs) and other safety results were collected. All AEs, serious and nonserious, observed in pwCF exposed to ivacaftor during this study were collected. Pulmonary exacerbations were collected as an effectiveness outcome; in this study, PEx was defined as worsening of the clinical state requiring acute antibiotics treatment and defined by the clinician as an exacerbation. PEx could also be spontaneously reported as an AE. The proportions of pwCF treated with ivacaftor presenting with abnormally high levels of serum aspartate aminotransferase (AST), alanine aminotransferase (ALT), $\gamma$-glutamyl transferase, alkaline phosphatase, and total bilirubin were assessed. Values for $\mathrm{ppFEV}_{1}$ were based on the Global Lung Function Initiative equations for White individuals [15].

All participant data were collected from paper or electronic medical records. Data were collected and transferred by local site staff onto a secure web-based electronic case report form. Data were collected retrospectively and/or prospectively for up to 12 months prior to ivacaftor initiation through up to 24 months after enrollment (Figure S1). Retrospective data were collected at study entry. Post-enrollment study data were collected approximately every 6 months for 2 years of participation. For pwCF already being treated with ivacaftor at study inclusion, the collection of on-treatment data was both retrospective and prospective. For those initiating ivacaftor at study inclusion, ontreatment data were only prospective. 


\section{Statistical Analysis}

Baseline value was defined as the last available value at or before the date of ivacaftor initiation. Analyses of lung function and nutritional status summarized the average changes from baseline within each 6-month interval after ivacaftor initiation. Analyses of clinical events evaluated rates in the 12 months prior to ivacaftor (pre-ivacaftor) compared with the 12 months after ivacaftor initiation (post-ivacaftor). Clinical events in the overall post-ivacaftor initiation period were summarized to provide an annualized rate.

A mixed model for repeated measures (MMRM) for continuous outcomes (e.g., $\mathrm{ppFEV}_{1}$ ) and a negative binomial model for PEx were used for analysis. Least squares (LS) means with 95\% CIs were obtained from an MMRM model, with the average change from baseline in a time interval as the dependent variable, categorical time interval as a fixed effect, continuous baseline value as a covariate, the pwCF as a random effect, and an unstructured covariance matrix for repeated measures. The estimated event rates and estimated number of days per person-year with 95\% CIs for the 12 months pre-ivacaftor and first 12 months postivacaftor, and the corresponding ratio (with 95\% CI) of 12 months post-ivacaftor relative to 12 months pre-ivacaftor, were obtained from a log-negative binomial regression model. The model specified the number of events in each time interval as the dependent variable, categorical time interval as a fixed effect, and continuous $\mathrm{ppFEV}_{1}$ at baseline as a covariate with an unstructured covariance matrix for repeated measures. The estimated annualized event rate with 95\% CI for the overall post-ivacaftor period was obtained from a similar negative binomial regression model for the number of events in the 12 months pre-ivacaftor period and the overall post-ivacaftor period. The CIs were nominal and did not control for multiplicity. All analyses were performed using SAS ${ }^{\circledR}$ version 9.4 (SAS Institute, Cary, NC, USA).

\section{Ethics}

This study was performed in accordance with the Helsinki Declaration of 1964, and its later amendments. Each investigator used International Society for Pharmacoepidemiology guidelines for good pharmacoepidemiology practice (2007) and good pharmacovigilance practices. In accordance with French regulatory requirements for observational studies, written informed consent was obtained from each pwCF or their parent or legal guardian, as appropriate, and assent was obtained from each child (if applicable).

\section{RESULTS}

\section{Population}

A total of 129 pwCF were enrolled at 35 tertiary CF centers in France from March 16, 2016, to December 2, 2019 (Table 1). Of the 57 CF centers in France contacted regarding participation in the study, most centers (35) participated; those that declined did so because they did not have any pwCF who met inclusion criteria. The mean age at ivacaftor initiation was 19.1 years (range, 2-64 years), and 53 pwCF (41.1\%) were $<12$ years of age (Table 2). Most pwCF $(64.3 \%[n=83])$ had a $G 551 D$ allele, and G551D/F508del was the most common CFTR genotype $(39.5 \%[n=51])$. The baseline severity of CF disease covered a broad range, with $\mathrm{ppFEV}_{1}$ ranging from 17.7 percentage points (pp) to $125.8 \mathrm{pp}$ (mean [SD], 75.22 [24.86] pp). The range of ivacaftor exposure was 20-92 months, with a mean (SD) of 52.7 (20.8) months.

Data for $\geq 24$ months of treatment with ivacaftor was available for 123 pwCF, and 120 participants had initiated ivacaftor prior to enrollment (Figure S2). Seven pwCF (5.4\%) discontinued ivacaftor after enrollment; five pwCF (3.9\%) withdrew after discontinuation of ivacaftor (participation in another interventional clinical study $[n=2]$, lung transplantation $[n=1]$, moving to another city $[n=1]$, and missed visit $[n=1])$; two pwCF discontinued ivacaftor but remained in the study (artificial 
Table 1 Participant disposition

\begin{tabular}{ll}
\hline & $n(\%)$ \\
\hline All enrolled people with CF & 129 \\
Completed study & $124(96.1)$ \\
Discontinued from study & $5(3.9)$ \\
Reason for discontinuation from study & $3(2.3)$ \\
Physician decision ${ }^{\text {a }}$ & $2(1.6)$ \\
Other ${ }^{\text {b }}$ & $9(7.0)$ \\
Started ivacaftor treatment at or after enrollment & $9(7.0)$ \\
Started ivacaftor 0 to 3 months after enrolling & $120(93.0)$ \\
Enrolled in study after starting ivacaftor treatment & $12(9.3)$ \\
Enrolled $>0$ to 6 months after starting ivacaftor & $14(10.9)$ \\
Enrolled $>6$ to 12 months after starting ivacaftor & $31(24.0)$ \\
Enrolled $>12$ to 24 months after starting ivacaftor & $7(5.4)$ \\
Enrolled $>24$ to 36 months after starting ivacaftor & $15(11.6)$ \\
Enrolled $>36$ to 48 months after starting ivacaftor & $41(31.8)$ \\
Enrolled $>48$ months after starting ivacaftor & $68(52.7)$ \\
Months of ivacaftor treatment completed (months) & $129(100.0)$ \\
$\geq 12$ & $123(95.3)$ \\
$\geq 24$ & $99(76.7)$ \\
$\geq 36$ &
\end{tabular}

$C F$ cystic fibrosis

${ }^{a}$ One person with CF underwent a bilateral lung transplantation. Two people with CF were included in a clinical study of cystic fibrosis transmembrane conductance regulator modulators

b One person with CF discontinued after moving to a different city. One person with CF was lost to follow-up

insemination $[n=1]$ and excessive weight gain $[n=1])$. Twenty-two pwCF $(17.1 \%)$ interrupted ivacaftor, with $\mathrm{AE}$ being the most common reason $(n=4[3.1 \%])$. Other reasons included the individual deciding not to take or forgetting to take ivacaftor, difficulties obtaining drug supply, and travel. Interruptions ranged from 1-177 days.

\section{Clinical Outcomes}

The LS mean (SE) ppFEV $_{1}$ increased by 8.49 (1.08) pp through the first 6 months of ivacaftor from a baseline mean (SD) of 75.22 (24.86) pp; this improvement was sustained through up to 36 months of ivacaftor (Fig. 1a).

The estimated event rates for all PEx were 0.86 events per person-year in the 12 months of the pre-ivacaftor period and 0.49 events per person-year in the first 12 months of the postivacaftor period; the associated estimated rate 
Table 2 Demographics and clinical characteristics at baseline

\begin{tabular}{|c|c|}
\hline Characteristic & $n=129$ \\
\hline Male sex, $n(\%)$ & $73(56.6)$ \\
\hline Age at IVA initiation, mean (range), years & $19.1(2-64)$ \\
\hline \multicolumn{2}{|l|}{ Age at IVA initiation category, $n(\%)$, years } \\
\hline$<6$ & $14(10.9)$ \\
\hline$\geq 6$ to $<12$ & $39(30.2)$ \\
\hline$\geq 12$ to $<18$ & $23(17.8)$ \\
\hline$\geq 18$ & $53(41.1)$ \\
\hline \multicolumn{2}{|l|}{ CFTR mutation for first allele, $n(\%)$} \\
\hline G551D/F508del & $51(39.5)$ \\
\hline$G 551 D /$ other $^{\mathrm{a}}$ & $32(24.8)$ \\
\hline$G 1244 E$ & $11(8.5)$ \\
\hline$S 1251 N$ & $11(8.5)$ \\
\hline G178R & $6(4.7)$ \\
\hline$S 549 N$ & $6(4.7)$ \\
\hline$S 549 R$ & $6(4.7)$ \\
\hline S1255P & $1(0.8)$ \\
\hline$G 1349 D$ & $1(0.8)$ \\
\hline R117H & $1(0.8)$ \\
\hline Other $^{\mathrm{b}}$ & $3(2.3)$ \\
\hline BMI-for-age $z$ score (age $<20$ years), mean (SD) & $-0.48(1.04) ; n=74^{\mathrm{c}}$ \\
\hline BMI (age $\geq 20$ years), mean $(S D)$ & $22.04(2.61) ; n=48^{\mathrm{c}}$ \\
\hline $\operatorname{ppFEV}_{1}$, mean $(S D)$, percentage points ${ }^{\mathrm{d}}$ & $75.2(24.9) ; n=115^{\mathrm{c}}$ \\
\hline \multicolumn{2}{|l|}{ ppFEV $_{1}$ severity, $n(\%)$} \\
\hline$<70 \%$ & $38(29.5)$ \\
\hline$\geq 70$ to $\leq 90 \%$ & $42(32.6)$ \\
\hline$>90 \%$ & $35(27.1)$ \\
\hline Missing & $14(10.9)$ \\
\hline
\end{tabular}

Baseline was the last available measurement on or prior to date of IVA initiation $B M I$ body mass index, $C F$ cystic fibrosis, $C F T R$ cystic fibrosis transmembrane conductance regulator, $I V A$ ivacaftor, $p p F E V_{1}$ percent predicted forced expiratory volume in $1 \mathrm{~s}, S D$ standard deviation

a The second allele was reported only for individuals with G551D-CFTR mutations

b One person with CF had genotype $G 541 D / W 1282 X$, one had $W 1282 X / D 1152 H$, and one had $R 347 H / 2183 A A-G$

c $n$ is the number of people with CF with available assessment

${ }^{d} \operatorname{ppFEV}_{1}$ values were based on the Global Lung Function Initiative equations for the White individuals [15] 

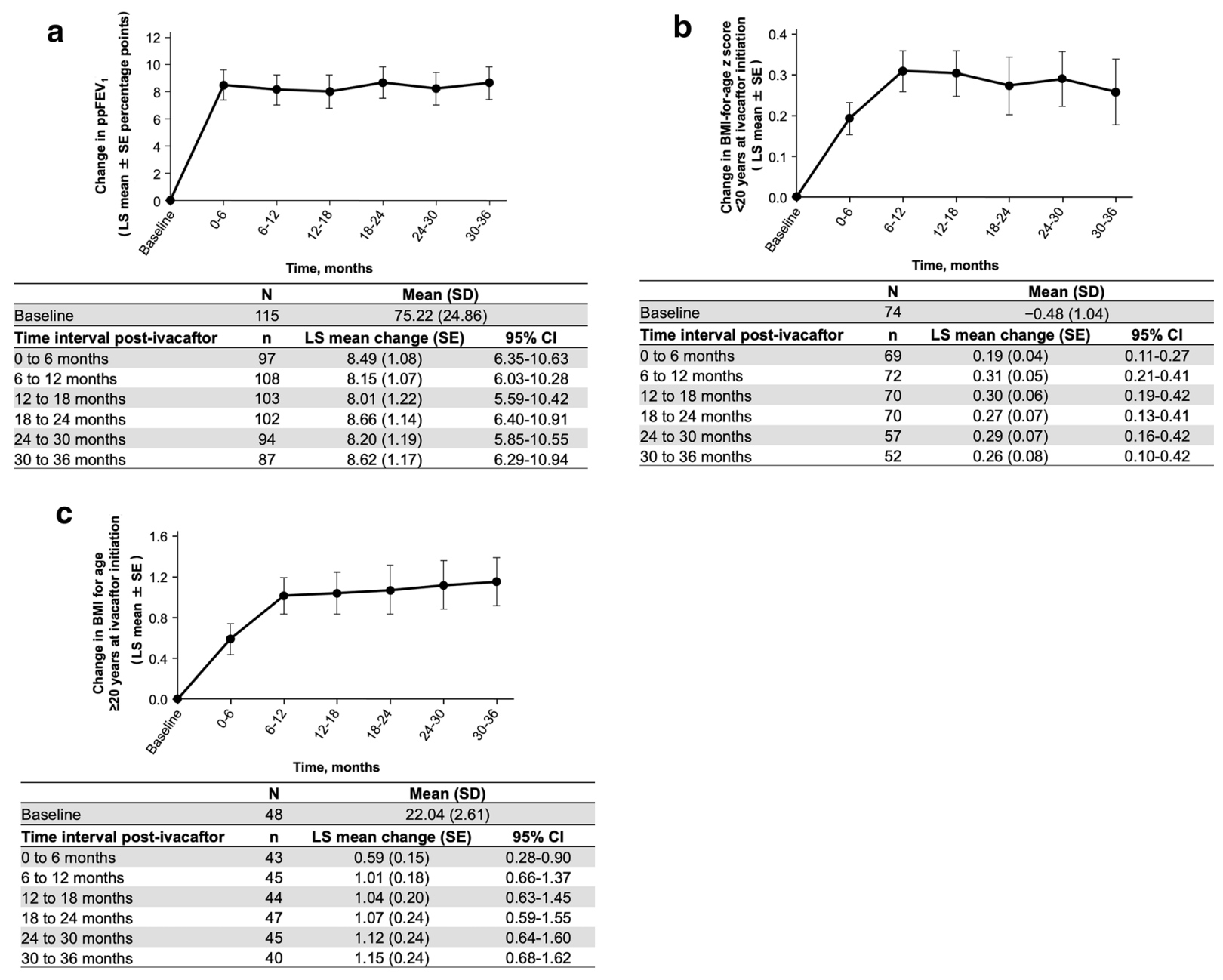

Fig. 1 Absolute change from baseline in a $\mathrm{ppFEV}_{1}$, b BMI-for-age $z$ score, and c BMI. BMI body mass index, $L S$ least squares, $p p F E V_{1}$ percent predicted forced expiratory volume in $1 \mathrm{~s}$

ratio was 0.57 (95\% CI $0.43-0.75)$. For the overall post-ivacaftor period (i.e., the rates that occurred during overall exposure to ivacaftor), the PEx event rate was 0.57 per person-year. In the 12 months of the pre-ivacaftor period, the number of days of PEx per person-year was 19.22 days and 10.92 days for the first 12 months on ivacaftor; the estimated rate ratio was 0.57 (95\% CI 0.43-0.75). The number of days of PEx was 12.46 days per person-year for the overall post-ivacaftor period (Table S1).

For PEx requiring hospitalization, the estimated event rate decreased from 0.11 events per person-year in the 12 months of the pre-ivacaftor period to 0.03 events per person-year during the first 12 months of the post-ivacaftor period; the associated estimated rate ratio was 0.25 (95\% CI 0.13-0.48). The event rate of PEx requiring hospitalization was 0.06 events per person-year during the overall post-ivacaftor period (Table 3).

For pwCF aged $<20$ years at ivacaftor initiation $(n=79)$, the mean (SD) BMI-for-age $Z$ score was -0.48 (1.04) at baseline; the LS mean (SE) $z$ score increased by $0.31(0.05)$ during the first 12 months following ivacaftor initiation and then remained relatively stable (Fig. 1b). In pwCF aged $\geq 20$ years at ivacaftor initiation, the mean (SD) BMI at baseline was $22.04(2.61) \mathrm{kg} / \mathrm{m}^{2}$; the LS mean 
Table 3 Number of exacerbations, hospitalizations, and courses of medication before and post ivacaftor treatment

\begin{tabular}{|c|c|c|c|}
\hline & $\begin{array}{l}\text { Pre-ivacaftor } \\
-12 \text { to } 0 \text { months } \\
(n=129)\end{array}$ & $\begin{array}{l}\text { Post-ivacaftor } \\
>0 \text { to } 12 \text { months } \\
(n=129)\end{array}$ & $\begin{array}{l}\text { Post-ivacaftor }^{a} \\
\text { Overall } \\
(n=129)\end{array}$ \\
\hline \multicolumn{4}{|l|}{ PEx } \\
\hline \multicolumn{4}{|l|}{ PEx ${ }^{b}$} \\
\hline Events, $n$ & 114 & 63 & 372 \\
\hline pwCF with events, N1 & 55 & 44 & 78 \\
\hline $\begin{array}{l}\text { Estimated annualized event rate per person with } \mathrm{CF}^{c} \\
(95 \% \mathrm{CI})\end{array}$ & $0.86(0.66-1.13)$ & $0.49(0.36-0.66)$ & $0.57(0.44-0.73)$ \\
\hline Estimated rate ratio $(95 \% \mathrm{CI})$ & & $0.57(0.43-0.75)$ & \\
\hline \multicolumn{4}{|l|}{ PEx requiring hospitalization ${ }^{\mathrm{b}}$} \\
\hline Events, $n$ & 24 & 7 & 56 \\
\hline pwCF with events, $n$ & 15 & 7 & 22 \\
\hline $\begin{array}{l}\text { Estimated annualized event rate per person with } \mathrm{CF}^{\mathrm{c}} \\
(95 \% \mathrm{CI})\end{array}$ & $0.11(0.04-0.27)$ & $0.03(0.01-0.08)$ & $0.06(0.03-0.13)$ \\
\hline Estimated rate ratio $(95 \% \mathrm{CI})$ & & $0.25(0.13-0.48)$ & \\
\hline \multicolumn{4}{|l|}{ Hospitalizations } \\
\hline \multicolumn{4}{|l|}{ Hospitalizations (all causes) ${ }^{\mathrm{b}}$} \\
\hline Events, $n$ & 70 & 34 & 155 \\
\hline pwCF with events, $n$ & 38 & 24 & 51 \\
\hline $\begin{array}{l}\text { Estimated annualized event rate per person with } \mathrm{CF}^{c} \\
(95 \% \mathrm{CI})\end{array}$ & $0.47(0.33-0.67)$ & $0.22(0.15-0.33)$ & $0.22(0.16-0.31)$ \\
\hline Estimated rate ratio $(95 \% \mathrm{CI})$ & & $0.48(0.32-0.71)$ & \\
\hline \multicolumn{4}{|l|}{ Medications } \\
\hline \multicolumn{4}{|l|}{ Courses of all acute antibiotics due to $\mathrm{PEx} \mathrm{x}^{\mathrm{b}}$} \\
\hline Courses, $n$ & 204 & 106 & 638 \\
\hline pwCF using antibiotics, $n$ & 52 & 45 & 79 \\
\hline $\begin{array}{l}\text { Estimated annualized course rate per person with } \mathrm{CF}^{\mathrm{c}} \\
(95 \% \mathrm{CI})\end{array}$ & $1.43(1.06-1.93)$ & $0.79(0.58-1.07)$ & $0.88(0.65-1.20)$ \\
\hline Estimated rate ratio $(95 \% \mathrm{CI})$ & & $0.55(0.40-0.75)$ & \\
\hline \multicolumn{4}{|l|}{ Courses of acute IV antibiotics due to PEx ${ }^{\mathrm{b}}$} \\
\hline Courses, $n$ & 88 & 32 & 178 \\
\hline pwCF using IV antibiotics, $n$ & 23 & 12 & 27 \\
\hline
\end{tabular}


Table 3 continued

\begin{tabular}{clll}
\hline & $\begin{array}{c}\text { Pre-ivacaftor } \\
-\mathbf{1 2} \text { to } \mathbf{0} \text { months } \\
(\boldsymbol{n}=\mathbf{1 2 9})\end{array}$ & $\begin{array}{l}\text { Post-ivacaftor } \\
>\mathbf{0} \text { to 12 } \text { months } \\
(\boldsymbol{n}=\mathbf{1 2 9})\end{array}$ & $\begin{array}{l}\text { Post-ivacaftor }^{\mathbf{a}} \\
\text { Overall }_{(\boldsymbol{n}=129)}\end{array}$ \\
\hline $\begin{array}{c}\text { Estimated annualized course rate per } \\
\text { person with } \mathrm{CF}^{\mathrm{c}}(95 \% \mathrm{CI})\end{array}$ & $0.45(0.26-0.78)$ & $0.18(0.09-0.36)$ & $0.15(0.09-0.27)$ \\
Estimated rate ratio $(95 \% \mathrm{CI})$ & & $\mathbf{0 . 3 9}(\mathbf{0 . 2 3 - 0 . 6 6 )}$ & \\
\hline
\end{tabular}

$C F$ cystic fibrosis, $I V$ intravenous, $P E x$ pulmonary exacerbation, $p w C F$ people with cystic fibrosis

a The post-ivacaftor $>0$ months period includes the entire follow-up period of pwCF while receiving ivacaftor treatment and was calculated using the exposure per pwCF as the denominator

${ }^{\mathrm{b}}$ Events with start date in interval were analyzed. PwCF could be counted in $>1$ interval for the same event

${ }^{c}$ Event rate per person-year was the number of events divided by total time on study in person-years within a time interval

(SE) of BMI increased by $1.01(0.18) \mathrm{kg} / \mathrm{m}^{2}$ during the first 12 months following ivacaftor initiation and was sustained through 36 months of ivacaftor (Fig. 1c).

\section{Healthcare Resource Utilization}

The estimated event rate for all hospitalizations (regardless of cause) was 0.47 hospitalizations per person-year in the 12 months of the pre-ivacaftor and 0.22 hospitalizations per person-year in the first 12 months of the post-ivacaftor period; the associated estimated rate ratio was 0.48 (95\% CI $0.32-0.71$ ). For the overall post-ivacaftor period, the event rate of hospitalizations was 0.22 per person-year (Table 3). The estimated number of days of hospitalization per person-year was 3.65 days for the 12-months pre-ivacaftor period and 1.43 days for the first 12 months on ivacaftor. The estimated rate ratio was 0.39 (95\% CI 0.24-0.63). The number of days of hospitalization was 1.58 days per person-year for the overall post-ivacaftor period (Table S1).

The estimated number of antibiotics courses per person-year was 1.43 courses during the preivacaftor period and 0.79 courses during the first 12 months of the post-ivacaftor period; the associated estimated rate ratio was 0.55 (95\% CI $0.40-0.75)$. For the overall post-ivacaftor period, the rate was 0.88 courses per person-year (Table 3). For IV antibiotics, the estimated rate ratio for the first 12 months after ivacaftor initiation vs the 12 months pre-ivacaftor was 0.39 (95\% CI 0.23-0.66). Similar trends were seen for numbers of days of antibiotics use for PEx (Table S1).

\section{Pulmonary Microbiology}

The percentage of pwCF with a positive culture for $\geq 1$ of the assessed respiratory pathogens was higher during the 12 months of the preivacaftor period than during the first 48 months of the overall post-ivacaftor period $(90.9 \%$ of pwCF pre-ivacaftor and a range of $75.8 \%$ to $83.3 \%$ annually of pwCF post-ivacaftor; Figure S3). Methicillin-sensitive Staphylococcus aureus, Pseudomonas aeruginosa, Aspergillus fumigatus, and Haemophilus influenzae were the most frequently identified pathogens in positive cultures. Stenotrophomonas maltophilia and methicillin-resistant $S$. aureus were less common, while Achromobacter xylosoxidans, nontuberculous mycobacteria, and Burkholderia cepacia complex were positive in $<4 \%$ of pwCF. The prevalence of a positive culture of methicillin-sensitive $S$. aureus and $P$. aeruginosa in pwCF was higher during the 12 months of the pre-ivacaftor period than during the first 12 months of the overall post-ivacaftor period (58.2\% vs. $49.6 \%$ and $48.2 \%$ vs. $33.9 \%$, respectively); the percentage of pwCF with a positive culture for these two organisms generally remained lower throughout the first 48 months of the overall post-ivacaftor period. For the other pathogens cultured, prevalence was variable or too low to draw conclusions about changes over time (Figure S3). 


\section{Safety}

Overall, 252 AEs were reported in 75 pwCF (58.1\%) (Table S2). Six pwCF (4.7\%) had AEs (serious or nonserious) considered by the investigator to be related to ivacaftor, including weight increase $(n=4)$, hemorrhage (cheek bleeding; $n=1)$, and rash $(n=1)$. Of the 252 AEs, 47 serious AEs were reported in 25 pwCF (19.4\%). The most common serious AEs were PEx $(n=3$ [2.3\%]; three events), hemoptysis ( $n=2$ [1.6\%]; five events), and influenza ( $n=2$ [1.6\%]; two events). Most serious AEs were individual cases reported in only 1 pwCF. One serious $\mathrm{AE}$ was reported to be related to ivacaftor; the case of hemorrhage was further described as "cheek bleeding" by the investigator, and the event was reported as resolved 1 day after onset. No deaths were reported.

\section{Hepatic Enzymes}

One pwCF had hepatitis with highly elevated $\operatorname{ALT}(>8 \times$ upper limit of normal [ULN]) and AST $(>8 \times$ ULN $)$ while taking ivacaftor (Table S3). This individual recovered within 2 weeks and continued ivacaftor for $>4$ years with no recurrence of signs of hepatitis. No other pwCF had ALT or AST $>8 \times$ ULN.

\section{Other Endpoints}

The most commonly reported comorbidities were exocrine pancreatic insufficiency, gastroesophageal reflux disease, nasal polyps, and CFrelated diabetes. Little difference was observed in prevalence of these comorbidities between the pre- and post-ivacaftor periods. One pwCF underwent pulmonary transplantation $>5$ years after ivacaftor initiation. The individual discontinued ivacaftor and withdrew from the study. No notable changes in chronic medication use or pancreatic enzyme use occurred with ivacaftor (data not shown).

\section{DISCUSSION}

Here we report on ivacaftor use in a real-world setting in France. The main results were substantial improvements in $\operatorname{ppFEV}_{1}$, growth metrics, and nutritional status after initiating ivacaftor. These were observed early within the first 6 months following ivacaftor initiation and sustained through up to 36 months of treatment. The changes observed were consistent with the previously reported improvements of $5.0-13.5 \mathrm{pp}$ in $\mathrm{ppFEV}_{1}, 0.30-1.5 \mathrm{~kg} / \mathrm{m}^{2}$ in BMI, and $0.4-0.45$ in BMI-for-age $z$ score in clinical studies of ivacaftor and in real-world observational and registry studies [7, 8, 16-19]. The

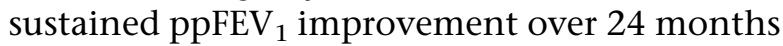
is highly relevant since progressive lung damage is normally observed in CF [20]. The improvement in growth metrics and nutritional status observed in both age groups (aged $<20$ years and $\geq 20$ years) is important contrasted with the poor growth trajectory commonly observed in pwCF $[21,22]$, and optimized growth metrics and nutritional status are well-known factors for improving survival in pwCF.

Treatment with ivacaftor was associated with a reduction of $43 \%$ for both annualized incidence and number of days of PEx, and it was associated with a $75 \%$ reduction of annualized incidence of PEx requiring hospitalization in accordance with previous reports [18, 19]. Interestingly, we also observed a decrease in the annual percentage of pwCF who had a positive respiratory culture after initiating ivacaftor. We observed a lower annual prevalence of infections of two main pathogens (methicillin-sensitive $S$. aureus and $P$. aeruginosa) after initiation of ivacaftor. These microbiology results supported previous observations [16, 17, 23]. $S$. aureus and $P$. aeruginosa infections are associated with poor outcomes and increase treatment burden, often requiring repeated antibiotics courses [24]. Our findings are congruent with observations made in a recently published 5-year follow-up of a US cohort of pwCF treated with ivacaftor [25]; the current study enrolled a larger population, including children as young as 2 years of age, but had a shorter follow-up duration. Taken together, these results are 
highly clinically relevant and further support the benefits of ivacaftor and the potential to alter the clinical course of CF disease.

Monitoring of hepatic safety in this population did not reveal any untoward findings in a real-world setting. Only one person reported highly elevated hepatic enzymes while taking ivacaftor, albeit transitorily. The individual recovered and continued ivacaftor for $>4$ years with no recurrence of liver disease. Overall, adverse event data were generally consistent with underlying CF disease and complemented the known safety profile of ivacaftor $[7,8,18,19,26-28]$, and no deaths occurred in this study.

This study comprised the largest population of pwCF in France receiving ivacaftor to date; it included pwCF with genotypes beyond G551D and followed them for the longest duration of real-world ivacaftor treatment. This is the first study of ivacaftor with real-life data in French children $<12$ years of age $(\sim 40 \%$ of participants). While this study was designed to include 24 months of prospective data for each pwCF, $76.7 \%$ of pwCF were exposed to $>36$ months of ivacaftor. This apparent discrepancy can be explained by the inclusion of retrospective data from those already receiving ivacaftor.

In this observational study, the participating population was more heterogeneous than a trial population, and the baseline severity of CF disease was broad. As such, these results provide important data from outside of clinical trials and are reflective of the standard of care in France.

\section{Limitations}

A limitation of this study was the enrollment of pwCF who were initiating ivacaftor and pwCF already receiving ivacaftor at enrollment; thus, data were analyzed relative to date of ivacaftor initiation rather than study entry date. Consequently, results beyond 24 months should be interpreted with caution. Additionally, incompleteness of pre-enrollment data is an inherent limitation of observational studies that collect retrospective data from medical files, which however were mainly electronic. Also, lack of a comparator cohort did not allow controlling for changes over time due to natural disease progression. In this observational study, a PEx was defined as a worsening of the clinical state requiring acute antibiotics treatment and defined by the clinician as an exacerbation. In the ivacaftor clinical trials, a PEx was defined as a requirement for new or changed antibiotic therapy (IV, inhaled, or oral) for $\geq 4$ signs and symptoms $[8,19,29]$; due to these differences in collection of PEx outcomes, direct comparisons should not be made between this observational study and other clinical studies. The pwCF enrolled in this real-world observational study were from a single country; thus caution must be exercised in extrapolating findings to other parts of the world due to differences in standards of care and health systems.

\section{CONCLUSIONS}

This observational study demonstrated the clinical effectiveness and safety of ivacaftor in a real-world setting in France. In addition, ivacaftor was associated with a reduction in HCRU. These data suggest that treatment with CFTR modulators can modify the outcomes of CF disease in pwCF with indicated mutations.

\section{ACKNOWLEDGEMENTS}

The authors thank all the people with CF who took part in the study and their families, as well as the principal investigators and research coordinators at each site. The authors thank all of the site investigators and clinical research associates who collected data at study sites.

Collaborators: BRIO Study Group: Sophie Ramel, Philippe Vigneron, Veronique Storni, Natacha Remus, Laurence Bassinet, Isabelle Durieu, Muriel Laurans, Cinthia Rames, Françoise Troussier, Marie-Laure Dalphin, Frédéric Huet, Isabelle Pin, Boubou Camara, Annabelle Payet, Jeanne Languepin, Isabelle Danner-Boucher, Marc Albertini, Julie Mankikian, Nathalie Wizla, Laure Cosson, Eric Deneuville, Marie Mittaine, Philippe Reix, Nadine Dufeu, Valérie David, Thierry Langin, Stéphanie Bui, Raphael 
Chiron, Dominique Grenet, Frédérique Chedevergne, Chantal Belleguic, and Laurent Mely.

Funding. This study was supported by Vertex Pharmaceuticals Incorporated. The sponsor was involved in the study design, analysis, and interpretation of the data, with collaboration from the authors. The sponsor helped develop the report with input, review, and approval from the authors. Vertex Pharmaceuticals Incorporated also funded the journal's Rapid Service Fee.

Medical Writing and/or Editorial Assistance. Editorial coordination and support were provided by Francesca Francois, PharmD, MPH, of Vertex Pharmaceuticals Incorporated; Francesca Francois may own stock or stock options in that company. Medical writing and editorial support were provided under the direction of the authors by JoAnna Anderson, PhD, CMPP, and Karen Kaluza Smith, PhD, CMPP. JoAnna Anderson and Karen Kaluza Smith are employees of ArticulateScience LLC, which received funding from Vertex Pharmaceuticals Incorporated.

Authorship. All named authors meet the International Committee of Medical Journal Editors (ICMJE) criteria for authorship for this article, take responsibility for the integrity of the work as a whole, and have given their approval for this version to be published.

Authors' Contributions. All authors contributed to data interpretation, conception, drafting, and/or revisions to the manuscript, and all approved the final version that was submitted for publication.

Prior Presentation. The final analysis of the BRIO study has not been previously presented or published. Interim analyses from this study have been previously presented at: 32 nd Annual North American Cystic Fibrosis Conference, October 18-20, 2018, Denver, CO, USA. ISPOR Europe 2018: New Perspectives for Improving 21st Century Health Systems, November 10-14, 2018, Barcelona, Spain. 23e Congrès de Pneumologie de Langue Française 2019 (23rd French
Language Pulmonology Congress 2019), January 25-27, 2019, Marseille, France.

Disclosures. All authors received nonfinancial support (assistance with manuscript preparation) from ArticulateScience LLC, which received funding from Vertex Pharmaceuticals Incorporated. Additional disclosures are as follows: Dominique Hubert discloses personal fees from Vertex Pharmaceuticals Incorporated. Christophe Marguet discloses personal fees and nonfinancial support from Vertex Pharmaceuticals Incorporated. Jacques Benichou has no additional disclosures. Cynthia DeSouza is a former employee of Vertex Pharmaceuticals Incorporated and may own stock or stock options in that company; she is currently an independent consultant. Catherine PayenChampenois, Nils Kinnman, and Keval Chandarana are employees of Vertex Pharmaceuticals Incorporated and may own stock or stock options in that company. Anne Munck discloses personal fees from Vertex Pharmaceuticals Incorporated. Isabelle Fajac discloses grants and personal fees from Vertex Pharmaceuticals Incorporated.

Compliance with Ethics Guidelines. This study was performed in accordance with the Helsinki Declaration of 1964, and its later amendments. Each investigator used International Society for Pharmacoepidemiology guidelines for good pharmacoepidemiology practice (2007) and good pharmacovigilance practices. This was an epidemiological observational study not modifying in any way the medical management of persons entering the study, not harming their physical or mental integrity, and not requiring any special monitoring visits. There were no additional or unusual diagnostic or surveillance procedures and no changes to the medications prescribed for the participants. Under these conditions, this study did not fall within the scope of French Research Planning Law 2006-450 of 18 April 2006 nor French Law 2004-806 of 09 August 2004, Article 88, Chapter II, Article L.1121-1, and the project, therefore, did not require submission to the Agence Nationale de Sécurité du Médicament et des Produits de Santé or the 
Ethics Committee. Written informed consent was obtained from each pwCF or their parent or legal guardian, as appropriate, and assent was obtained from each child (if applicable).

Data Availability. Vertex Pharmaceuticals Incorporated is committed to advancing medical science and improving the health of people with cystic fibrosis. This includes the responsible sharing of clinical trial data with qualified researchers. Proposals for the use of these data will be reviewed by a scientific board. Approvals are at the discretion of Vertex Pharmaceuticals Incorporated and will be dependent on the nature of the request, the merit of the research proposed, and the intended use of the data. Please contact CTDS@vrtx.com if you would like to submit a proposal or need more information.

Open Access. This article is licensed under a Creative Commons Attribution-NonCommercial 4.0 International License, which permits any non-commercial use, sharing, adaptation, distribution and reproduction in any medium or format, as long as you give appropriate credit to the original author(s) and the source, provide a link to the Creative Commons licence, and indicate if changes were made. The images or other third party material in this article are included in the article's Creative Commons licence, unless indicated otherwise in a credit line to the material. If material is not included in the article's Creative Commons licence and your intended use is not permitted by statutory regulation or exceeds the permitted use, you will need to obtain permission directly from the copyright holder. To view a copy of this licence, visit http:// creativecommons.org/licenses/by-nc/4.0/.

\section{REFERENCES}

1. Van Goor F, Hadida S, Grootenhuis PD, Burton B, Cao D, Neuberger $T$, et al. Rescue of CF airway epithelial cell function in vitro by a CFTR potentiator, VX-770. Proc Natl Acad Sci USA. 2009;106(44):18825-30. https://doi.org/10.1073/ pnas.0904709106.
2. Van Goor F, Yu H, Burton B, Hoffman BJ. Effect of ivacaftor on CFTR forms with missense mutations associated with defects in protein processing or function. J Cyst Fibros. 2014;13(1):29-36. https:// doi.org/10.1016/j.jcf.2013.06.008.

3. Yu H, Burton B, Huang CJ, Worley J, Cao D, Johnson JP Jr, et al. Ivacaftor potentiation of multiple CFTR channels with gating mutations. J Cyst Fibros. 2012;11(3):237-45. https://doi.org/10.1016/j.jcf. 2011.12.005.

4. European Medicines Agency. Summary of opinion (initial authorisation). https://www.ema.europa.eu/ en/documents/smop-initial/chmp-summarypositive-opinion-kalydeco_en.pdf (2012). Accessed Oct 5, 2020.

5. Haute Autorité de Santé. Transparency committee: opinion. https://www.has-sante.fr/upload/docs/ application/pdf/2013-05/kalydeco_ct_12474.pdf.

Accessed Oct 5, 2020.

6. Gramegna A, Contarini M, Aliberti S, Casciaro R, Blasi F, Castellani C. From ivacaftor to triple combination: a systematic review of efficacy and safety of CFTR modulators in people with cystic fibrosis. Int J Mol Sci. 2020. https://doi.org/10.3390/ ijms21165882.

7. Davies JC, Wainwright CE, Canny GJ, Chilvers MA, Howenstine MS, Munck A, et al. Efficacy and safety of ivacaftor in patients aged 6 to 11 years with cystic fibrosis with a $G 551 D$ mutation. Am J Respir Crit Care Med. 2013;187(11):1219-25. https://doi.org/ 10.1164/rccm.201301-0153OC.

8. Ramsey BW, Davies J, McElvaney NG, Tullis E, Bell SC, Drevinek P, et al. A CFTR potentiator in patients with cystic fibrosis and the G551D mutation. N Engl J Med. 2011;365(18):1663-72. https://doi. org/10.1056/NEJMoa1105185.

9. European Medicines Agency. Summary of opinion (post authorisation). https://www.ema.europa. eu/en/documents/smop/chmp-post-authorisationsummary-positive-opinion-kalydeco_en.pdf (2014). Accessed Oct 5, 2020.

10. Haute Autorité de Santé. Transparency committee: opinion. https://www.has-sante.fr/upload/docs/ application/pdf/2015-09/kalydeco_en_ct13972_val. pdf. Accessed Oct 5, 2020.

11. European Medicines Agency. Assessment report. https://www.ema.europa.eu/en/documents/varia tion-report/kalydeco-h-c-2494-ii-0027-epar-assess ment-report-variation_en.pdf (2015). Accessed Oct 5,2020 .

12. European Medicines Agency. Assessment report. https://www.ema.europa.eu/en/documents/ 
variation-report/kalydeco-h-c-2494-x-0034-g-eparassessment-report-extension_en.pdf (2015). Accessed Oct 5, 2020.

13. Kalydeco (ivacaftor). Summary of product characteristics. Vertex Pharmaceuticals (Ireland) Limited; 2020. https://www.ema.europa.eu/documents/productinformation/kalydeco-epar-product-information en.pdf.

14. Centers for Disease Control and Prevention. Clinical growth charts. https://www.cdc.gov/ growthcharts/percentile_data_files.htm (2009). Accessed Dec 21, 2020.

15. Quanjer PH, Stanojevic S, Cole TJ, Baur X, Hall GL, Culver $\mathrm{BH}$, et al. Multi-ethnic reference values for spirometry for the 3-95-yr age range: the global lung function 2012 equations. Eur Respir J. 2012;40(6):1324-43. https://doi.org/10.1183/ 09031936.00080312 .

16. Hubert D, Dehillotte C, Munck A, David V, Baek J, Mely L, et al. Retrospective observational study of French patients with cystic fibrosis and a Gly551Asp-CFTR mutation after 1 and 2 years of treatment with ivacaftor in a real-world setting. J Cyst Fibros. 2018;17(1):89-95. https://doi.org/10. 1016/j.jcf.2017.07.001.

17. McKone EF, Borowitz D, Drevinek P, Griese $M$, Konstan MW, Wainwright C, et al. Long-term safety and efficacy of ivacaftor in patients with cystic fibrosis who have the Gly551Asp-CFTR mutation: a phase 3, open-label extension study (PERSIST). Lancet Respir Med. 2014;2(11):902-10. https://doi.org/10.1016/S2213-2600(14)70218-8.

18. De Boeck K, Munck A, Walker S, Faro A, Hiatt P, Gilmartin $G$, et al. Efficacy and safety of ivacaftor in patients with cystic fibrosis and a non-G551D gating mutation. J Cyst Fibros. 2014;13(6):674-80. https://doi.org/10.1016/j.jcf.2014.09.005.

19. Moss RB, Flume PA, Elborn JS, Cooke J, Rowe SM, McColley SA, et al. Efficacy and safety of ivacaftor in patients with cystic fibrosis who have an Arg117His-CFTR mutation: a double-blind, randomised controlled trial. Lancet Respir Med. 2015;3(7):524-33. https://doi.org/10.1016/S22132600(15)00201-5.

20. Harun SN, Wainwright C, Klein K, Hennig S. A systematic review of studies examining the rate of lung function decline in patients with cystic fibrosis. Paediatr Respir Rev. 2016;20:55-66. https://doi. org/10.1016/j.prrv.2016.03.002.

21. Bell SC, Bowerman AR, Davies CA, Campbell IA, Shale DJ, Elborn JS. Nutrition in adults with cystic fibrosis. Clin Nutr. 1998;17(5):211-5. https://doi. org/10.1016/S0261-5614(98)80061-7.
22. Yen EH, Quinton H, Borowitz D. Better nutritional status in early childhood is associated with improved clinical outcomes and survival in patients with cystic fibrosis. J Pediatr. 2013;162(3):530-5. https://doi.org/10.1016/j.jpeds.2012.08.040 (e1).

23. Rowe SM, Heltshe SL, Gonska T, Donaldson SH, Borowitz D, Gelfond D, et al. Clinical mechanism of the cystic fibrosis transmembrane conductance regulator potentiator ivacaftor in G551D-mediated cystic fibrosis. Am J Respir Crit Care Med. 2014;190(2):175-84. https://doi.org/10.1164/rccm. 201404-0703OC.

24. Limoli DH, Hoffman LR. Help, hinder, hide and harm: what can we learn from the interactions between Pseudomonas aeruginosa and Staphylococcus aureus during respiratory infections? Thorax. 2019;74(7):684-92. https://doi.org/10.1136/ thoraxjnl-2018-212616.

25. Guimbellot JS, Baines A, Paynter A, Heltshe SL, VanDalfsen J, Jain $M$, et al. Long-term clinical effectiveness of ivacaftor in people with the G551D CFTR mutation. J Cyst Fibros. 2020. https://doi.org/ 10.1016/j.jcf.2020.11.008 (Published online November 25).

26. Davies JC, Cunningham S, Harris WT, Lapey A, Regelmann WE, Sawicki GS, et al. Safety, pharmacokinetics, and pharmacodynamics of ivacaftor in patients aged 2-5 years with cystic fibrosis and a CFTR gating mutation (KIWI): an open-label, single-arm study. Lancet Respir Med. 2016;4(2): 107-15. 2600(15)00545-7.

27. Davies JC, Wainwright CE, Sawicki GS, Higgins MN, Campbell D, Harris C, et al. Ivacaftor in infants aged 4 to $<12$ months with cystic fibrosis and a gating mutation: results of a 2-part phase 3 clinical trial. Am J Respir Crit Care Med. 2020. https://doi. org/10.1164/rccm.202008-3177OC online October 7).

(Published

28. Rosenfeld M, Wainwright CE, Higgins M, Wang LT, McKee C, Campbell D, et al. Ivacaftor treatment of cystic fibrosis in children aged 12 to less than 24 months and with a CFTR gating mutation (ARRIVAL): a phase 3 single-arm study. Lancet Respir Med. 2018;6(7):545-53. https://doi.org/10.1016/ S2213-2600(18)30202-9.

29. Davies J, Sheridan H, Bell N, Cunningham S, Davis SD, Elborn JS, et al. Assessment of clinical response to ivacaftor with lung clearance index in cystic fibrosis patients with a G551D-CFTR mutation and preserved spirometry: a randomised controlled trial. Lancet Respir Med. 2013;1(8):630-8. http:// pubmed.ncbi.nlm.nih.gov/24461666/ 